
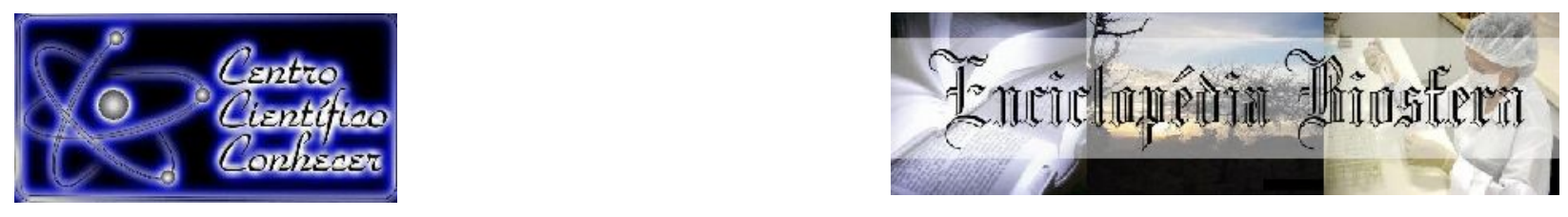

\title{
ESTUDO DA INTERFERÊNCIA DO RAIO DE PONTA DA FERRAMENTA SOBRE A RUGOSIDADE SUPERFICIAL EM UM PROCESSO DE TORNEAMENTO
}

\footnotetext{
Ithyara Dheylle Machado de Medeiros ${ }^{1}$, Aleksandro Guedes de Lima ${ }^{2}$, Laurivan da Silva Diniz ${ }^{2}$, Kelly Cristiane Gomes ${ }^{3}$, Aline Karla Barbosa da Silva ${ }^{4}$

${ }^{1}$ Doutoranda do Programa de Pós-Graduação em Engenharia Mecânica, UFPB João Pessoa - Brasil, ithyaramachado@gmail.com

${ }^{2}$ Professores do Departamento de Tecnologia em Automação Industrial do Instituto Federal da Paraíba - IFPB

${ }^{3}$ Professora do Departamento de Engenharia de Energias Alternativas e Renováveis

- DEER da Universidade Federal da Paraíba - UFPB

${ }^{4}$ Doutoranda do Programa de Pós-Graduação em Engenharia Mecânica da Universidade Federal da Paraíba - UFPB
}

Recebido em: 22/09/2018 - Aprovado em: 23/11/2018 - Publicado em: 03/12/2018 DOI: 10.18677/EnciBio_2018B114

\begin{abstract}
RESUMO
O torneamento de acabamento é caracterizado por ser uma operação de finalização da peça, em função do raio de ponta da ferramenta ser considerado um dos fatores de maior influência sobre a rugosidade superficial de peças que passaram pelo processo de torneamento de acabamento, aliado ao fato de pesquisas publicadas relatarem discrepâncias entre os cálculos teóricos e os métodos experimentais. O presente estudo teve como objetivo avaliar a interferência do raio de ponta da ferramenta em termos do parâmetro $\mathrm{Rz}$ medido, em relação ao esperado teoricamente, e analisar através de imagens tridimensionais e por vistas laterais, as marcas superficiais deixadas pela ferramenta de corte em cada uma das peças torneadas. A pesquisa permitiu identificar que ao aumentar o raio de ponta da ferramenta de $0,4 \mathrm{~mm}$ para $0,8 \mathrm{~mm}$ ocorreu uma diminuição da rugosidade superficial, em termos do parâmetro $\mathrm{Rz}$, quando o material torneado foi o alumínio, concordando com a equação teórica. No entanto, quando o material torneado foi o aço, o inverso ocorreu. Esse fato foi justificado em função da menor usinabilidade do aço e pelos outros parâmetros envolvidos no processo os quais não foram levados em consideração, tais como velocidade de corte, desgaste da ferramenta, atrito e forças de corte. Além disso, foram obtidas imagens por Microscopia óptica e por perfilometria (vistas laterais) que permitiram identificar as marcas de avanço e do raio de ponta da ferramenta, assim como irregularidades provenientes de fluxo lateral de cavaco, e do próprio atrito peça-ferramenta.
\end{abstract}

PALAVRAS-CHAVE: rugosidade, raio de ponta, torneamento.

\section{STUDY OF TIP RADIUS INTERFERENCE ON SURFACE ROUGHNESS IN A TURNING PROCESS}

\author{
ABSTRACT \\ Finishing turning is characterized by being a finishing operation of the workpiece, \\ depending on the tool radius being considered one of the factors that have the
}


greatest influence on the surface roughness of parts that have undergone the finishing turning process, together with the fact that published researches report discrepancies between theoretical calculations and experimental methods. The objective of the present study was to evaluate the interference of the tool tip radius in terms of the measured $R z$ parameter, in relation to what was theoretically expected, and to analyze, through three-dimensional images and lateral views, the surface marks left by the cutting tool in each one of turned parts. The research allowed to identify that when increasing the tip radius of the tool from $0.4 \mathrm{~mm}$ to $0.8 \mathrm{~mm}$ there was a decrease of the surface roughness, in terms of the parameter $R z$, when the material turned was aluminum, agreeing with the theoretical equation. However, when the material turned was steel, the reverse occurred. This fact was justified due to the lower machinability of the steel and the other parameters involved in the process which were not taken into account, such as cutting speed, tool wear, friction and shearing forces. In addition, images were obtained by optical microscopy and by profilometry (lateral views) that allowed to identify the marks of advance and the radius of tip of the tool, as well as irregularities coming from lateral flow of chip, and of the own friction-tool.

KEYWORDS: turning, roughness, tip radius.

\section{INTRODUÇÃO}

O torneamento de acabamento é caracterizado por ser uma operação de finalização da peça, dessa maneira, em muitas situações, esta é a única operação que estabelecerá a qualidade do produto usinado, uma vez que processos subsequentes apenas proporcionarão acréscimos aos custos desse (SOUZA et al., 2010).

Uma melhor qualidade superficial e longa vida útil dos produtos acabados são características que tem estimulado o progresso dos processos de usinagem (KUMAR et al., 2017). Conseguir um melhor acabamento de superfície com menor taxa de desgaste da ferramenta é o grande desafio liderado pelas indústrias de manufatura. Durante a usinagem, vários critérios devem ser satisfeitos, como economia de tempo, custo do material, e número de tentativas. Em tais situações, é difícil descobrir a melhor combinação possível das variáveis de corte com a satisfação de todos os critérios de saída. Para superar esse tipo de problema, é necessária a otimização dos parâmetros de corte (MAITY ; PRANDHAN, 2018).

Um bom entendimento do processo de remoção de material durante o corte é essencial para a seleção do material, projeto da ferramenta como também na garantia de precisão dimensional consistente e integridade da superfície do produto acabado. $O$ atrito gerado durante o processo influencia o poder de corte, a qualidade da usinagem, a vida útil da ferramenta e o custo de usinagem. Quando o desgaste da ferramenta atinge um determinado valor, aumentando a força de corte, vibração e temperatura de corte, ela causa deterioração da integridade da superfície e erro de dimensão maior que a tolerância (QEHAJA, 2015).

O erro de dimensão é preocupante, pois a rugosidade da superfície tem um efeito importante na vida de muitos componentes de engenharia. Baixos valores de rugosidade irão melhorar a vida à fadiga, reduzir o coeficiente de atrito e as taxas de desgaste e melhorar a resistência à corrosão e as propriedades estéticas. Por essas razões, o controle da rugosidade da superfície em componentes de engenharia está se tornando cada vez mais fundamental e isso se reflete em um uso mais difundido das especificações de rugosidade superficial nos desenhos técnicos. Muitas 
empresas, quando confrontadas com aspereza de superfície rigorosamente especificada, necessitam avaliar com mais cuidado seus procedimentos de acabamento de componentes. Um aspecto importante desta avaliação é uma tendência de utilizar um conjunto de parâmetros que proporcionem as melhores condições técnicas para atingir o nível necessário de rugosidade da superfície (GILLIBRAND et al., 1996).

Sabe-se que as condições impostas sobre a aresta de corte para uma operação de desbaste, durante o torneamento, apresentam divergências quando comparadas as impostas sobre uma aresta submetida ao procedimento de acabamento superficial. O desgaste da ferramenta durante o desbaste é decorrente, principalmente, de condições tais como: tipo de material usinado, tenacidade da ferramenta e taxa de remoção. Enquanto que no acabamento, os fatores mais importantes estão relacionados a resistência ao desgaste e a precisão da peça usinada (FERRARESI, 2014). Nesse sentido, o parâmetro "Rz" referente a rugosidade máxima é amplamente difundido em indústrias para caracterizar a qualidade de superfícies mecânicas após o torneamento de acabamento (HORVÁTH et al., 2015).

Segundo a Associação Brasileira de Normas Técnicas (2002), a rugosidade máxima ou altura máxima do perfil é definida como a soma da altura máxima dos picos do perfil $\mathrm{Zp}$ e a maior das profundidades dos vales do perfil $\mathrm{Zv}$, no comprimento de amostragem, como pode ser visualizado na Figura 1.

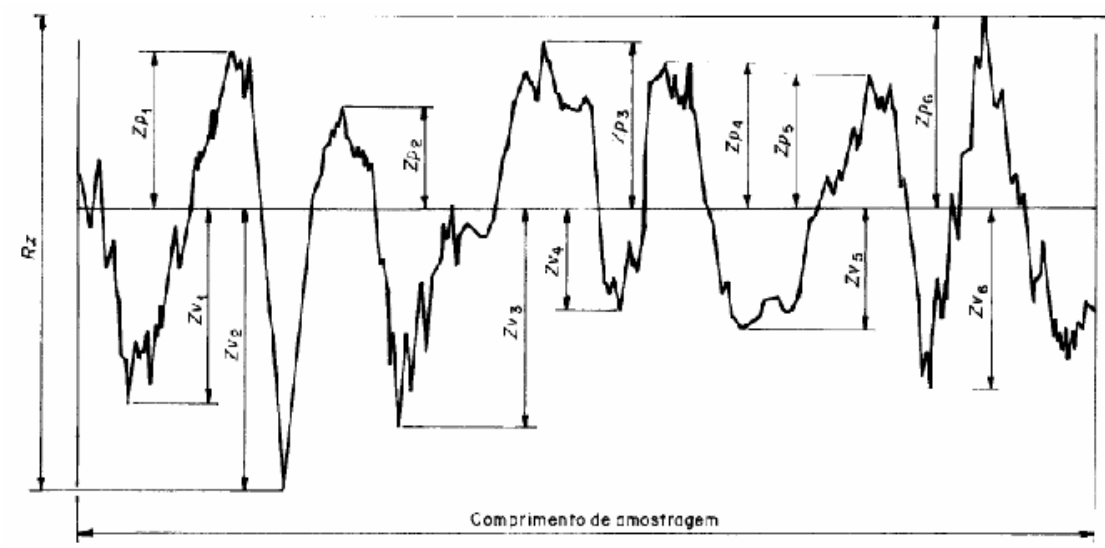

FIGURA 1 Rugosidade máxima (Rz) (ABNT, 2002).

O processo de torneamento de acabamento é usualmente executado sob condições que geram uma superfície proveniente da associação entre a aresta principal de corte, a secundária e o raio de ponta, originando uma região usinada que exibe um aspecto em função do raio de ponta (SOUZA et al., 2010), como pode ser compreendido por meio da visualização da Figura 2. 


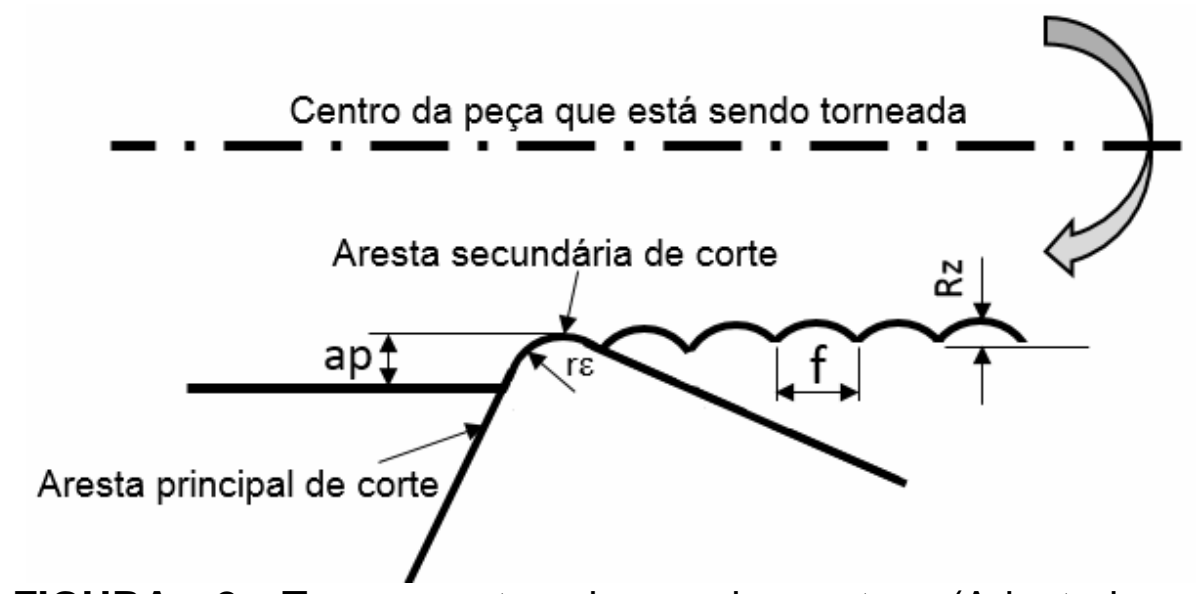

FIGURA 2 Torneamento de acabamento. (Adaptado de GILLIBRAND et al., 1996)

Quando o processo de torneamento é realizado em condições ideais, a distância entre o pico e o vale desta superfície representa a rugosidade máxima $(R z)$, como mostrado na Figura 2. Dessa forma, a equação 1 é descrita:

$$
R_{z}=\frac{f^{2}}{r_{g}}
$$

Onde:

$\mathrm{f}=$ avanço da ferramenta em milímetros por rotação, [mm/rot];

$r_{\varepsilon}=$ raio de ponta da ferramenta em milímetros, [mm];

No entanto, a Equação 1 só é verdadeira em condições ideias. Em função disso, vários são os estudos desenvolvidos (BHARDWAJ et al., 2014; ASILTÜRK et al., 2016; GRZESIK, 2018) que visam avaliar a influência desses, e dos demais parâmetros de usinagem envolvidos no processo de torneamento.

Grzesik (2018) procurou prever a rugosidade superficial (por meio do parâmetro $\mathrm{Rz}$ ) produzida por operações de torneamento de precisão utilizando ferramentas de corte de CBN e taxas de avanços variáveis de 0,025 a 0,075 $\mathrm{mm} / \mathrm{rev}$. As diferenças entre os parâmetros de rugosidade superficial e teórica foram quantificadas em termos de efeito de mola, suavização adicional de irregularidades e efeito de fluxo lateral. $O$ estudo experimental primário incluiu medições dos parâmetros de rugosidade da superfície 2D e 3D usando o perfilômetro de contato. Dessa forma, verificou-se que o valor medido da altura máxima da superfície difere do valor teórico principalmente devido à recuperação elástica da superfície usinada e o efeito de alisamento proveniente dos menores avanços, e a recuperação elástica e o efeito de fluxo lateral quando avanços maiores foram empregados.

No trabalho de Bhardwaj et al. (2014) uma tentativa foi feita para investigar a influência da velocidade de corte, avanço, profundidade de corte e raio da ferramenta na rugosidade da superfície durante o torneamento úmido do aço 353 usando pastilhas de metal duro. O estudo revelou que a velocidade foi o principal fator de influência na rugosidade da superfície, já a profundidade de corte não teve relevância sobre essa. O modelo matemático para a rugosidade superficial mostrou que ela diminui com o aumento da velocidade e do raio de ponta, mas aumenta com o aumento da alimentação. 
Asiltürk et al. (2016) buscaram determinar combinações de parâmetros experimentais ideais, fazendo uso de modelos de previsão, para obter os valores mínimos de $\mathrm{Rz}$ através de associações de parâmetros de usinagem. Para isso, variaram a rotação do torno (318, 477 e $636 \mathrm{rpm})$, o avanço $(0,1 ; 0,15$ e 0,25 $\mathrm{mm} / \mathrm{rot})$, a profundidade de corte $(0,5 ; 0,7$ e $0,9 \mathrm{~mm})$ e o raio de ponta da ferramenta $(0,4 ; 0,8$ e $1,2 \mathrm{~mm})$. Após os experimentos, determinaram que para o valor de $\mathrm{Rz} 0$ parâmetro mais significativo foi o raio de ponta da ferramenta com $43 \%$ e o avanço com 33\%.

Em razão do raio de ponta da ferramenta ser considerado um dos fatores de maior influência sobre a rugosidade superficial de peças que passaram pelo processo de torneamento externo, aliado ao fato da literatura (GRAVALOS et al., 2010; GRZESIK, 2018) mostrar discrepâncias entre os cálculos teóricos e os métodos experimentais. O presente estudo teve como objetivo avaliar a interferência do raio de ponta da ferramenta em termos do parâmetro $\mathrm{Rz}$ medido, em relação ao esperado teoricamente da Equação 1, e analisar através de imagens tridimensionais e por vistas laterais, as marcas superficiais deixadas pela ferramenta de corte em cada uma das peças torneadas.

\section{Material torneado}

\section{MATERIAL E MÉTODOS}

Os materiais utilizados para o torneamento foram aço AISI 1020 e alumínio. A partir desses materiais, foram torneadas quatro amostras, sendo duas de aço e duas de alumínio. Para concretizar o objetivo do presente estudo, foram cortados dois tarugos de cada tipo de material, conforme Figura 3.

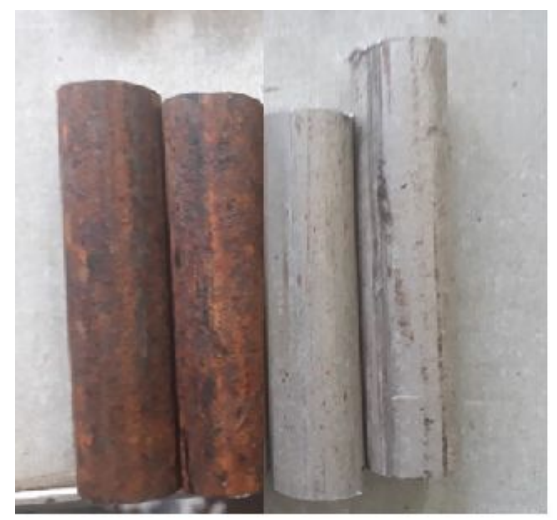

FIGURA 3 Materiais utilizados para o torneamento

\section{Parâmetros do torneamento} Quadro 1.

O presente estudo foi realizado utilizando os parâmetros de corte definidos no

QUADRO 1 Parâmetros de corte do presente estudo.

\begin{tabular}{ccc} 
Parâmetros & Abreviação & Valor adotado \\
\hline Velocidade de corte & Vc & $430 \mathrm{~m} / \mathrm{min}$ \\
\hline Rotação máxima & $\mathrm{n}$ & $3000 \mathrm{rpm}$ \\
\hline Profundidade de corte & ap & $0,3 \mathrm{~mm}$ \\
\hline Avanço & $\mathrm{f}$ & $0,22 \mathrm{~mm} / \mathrm{rot}$ \\
\hline
\end{tabular}




\section{Ferramenta de corte}

O torneamento foi realizado utilizando duas pastilhas de metal duro com raios de ponta diferentes a fim de comparação entre elas, sendo uma de 0,4 $\mathrm{mm}$ e outra de $0,8 \mathrm{~mm}$. A de 0,4 mm foi a SANDVIK Coromant DNMG $150604-$ PF 4225, e a de $0,8 \mathrm{~mm}$ foi a SANDVIK Coromant VBMT 160408 - PF 4225. As duas podem ser observadas na Figura 4.

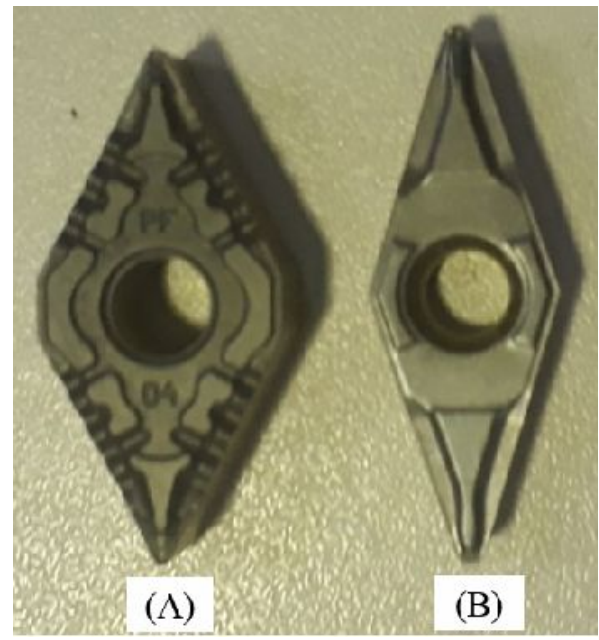

FIGURA 4 (A) pastilha DNMG 1506 04 - PF 4225; (B) pastilha VBMT 16 0408 - PF 4225.

\section{Nomenclatura das amostras}

Para efeito de melhor compreensão do tipo de material usinado e do raio de ponta da ferramenta empregados, as especificações das amostras estão listadas no Quadro 2.

QUADRO 2 Nomenclatura das amostras.

\begin{tabular}{ccc}
\hline Amostras & Material usinado & Raio de ponta [mm] \\
\hline Peça 1 & Aço & 0,4 \\
\hline Peça 2 & Aço & 0,8 \\
\hline Peça 3 & Alumínio & 0,4 \\
\hline Peça 4 & Alumínio & 0,8 \\
\hline
\end{tabular}

\section{Torno CNC}

O torno utilizado foi o Nardini Diplomat LOGIC 195 III, como pode ser visualizado na Figura 5.

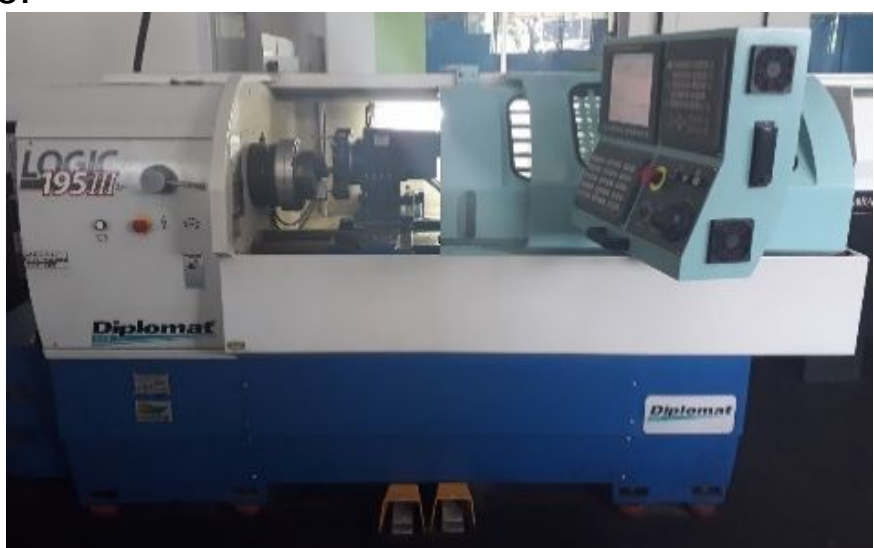

FIGURA 5 Torno CNC Nardini Diplomat LOGIC 195 III 


\section{Programação do torno}

A partir da programação FANUC, foi desenvolvido o seguinte programa para o torneamento das amostras do presente estudo:

N10 G21 G40 G90 G95;

N20 T0202;

N30 G54;

N40 G96 S430;

N50 G92 S3000 M03;

N60 G00 Z2;

N70 X19;

N80 G71 U1 R1;

N90 G71 P100 Q150 U0.3 W0.1 F0.2;

N100 G00 X0;

N110 G01 Z0;

N120 G01 X16;

N130 G01 Z-25;

N140 G01 X18;

N150 G01 Z-30;

N160 G42;

N170 G70 P100 Q150 F0.22;

N180 G40;

N190 M30.

\section{Técnicas de caracterização das peças torneadas}

A determinação da rugosidade superficial das peças torneadas foi obtida pelo aparelho de perfilometria óptica sem contato CCI MP, fabricado pela Taylor Hobson, conectado a uma unidade computadorizada contendo o software Talysurf $\mathrm{CCl}$ (Taylor Hobson, Inglaterra) para obtenção e análise dos dados. Em cada uma das peças torneadas foram realizadas sete leituras do parâmetro $R z$ da rugosidade. Essa leitura foi uniformemente espaçada ao longo de todo o comprimento da superfície torneada a fim de obter uma curva representativa da rugosidade.

A visualização das características morfológicas das peças torneadas foi obtida a partir do uso de um Microscópio Eletrônico de Varredura utilizando o princípio de funcionamento de elétrons secundários com módulo ambiental. $\mathrm{O}$ microscópio utilizado é do modelo Quanta 450, fabricado pela FEl.

\section{RESULTADOS E DISCUSSÃO}

O gráfico que ilustra a curva obtida por meio dos sete pontos de rugosidade medidas em cada uma das peças torneadas está ilustrado na Figura 6. 


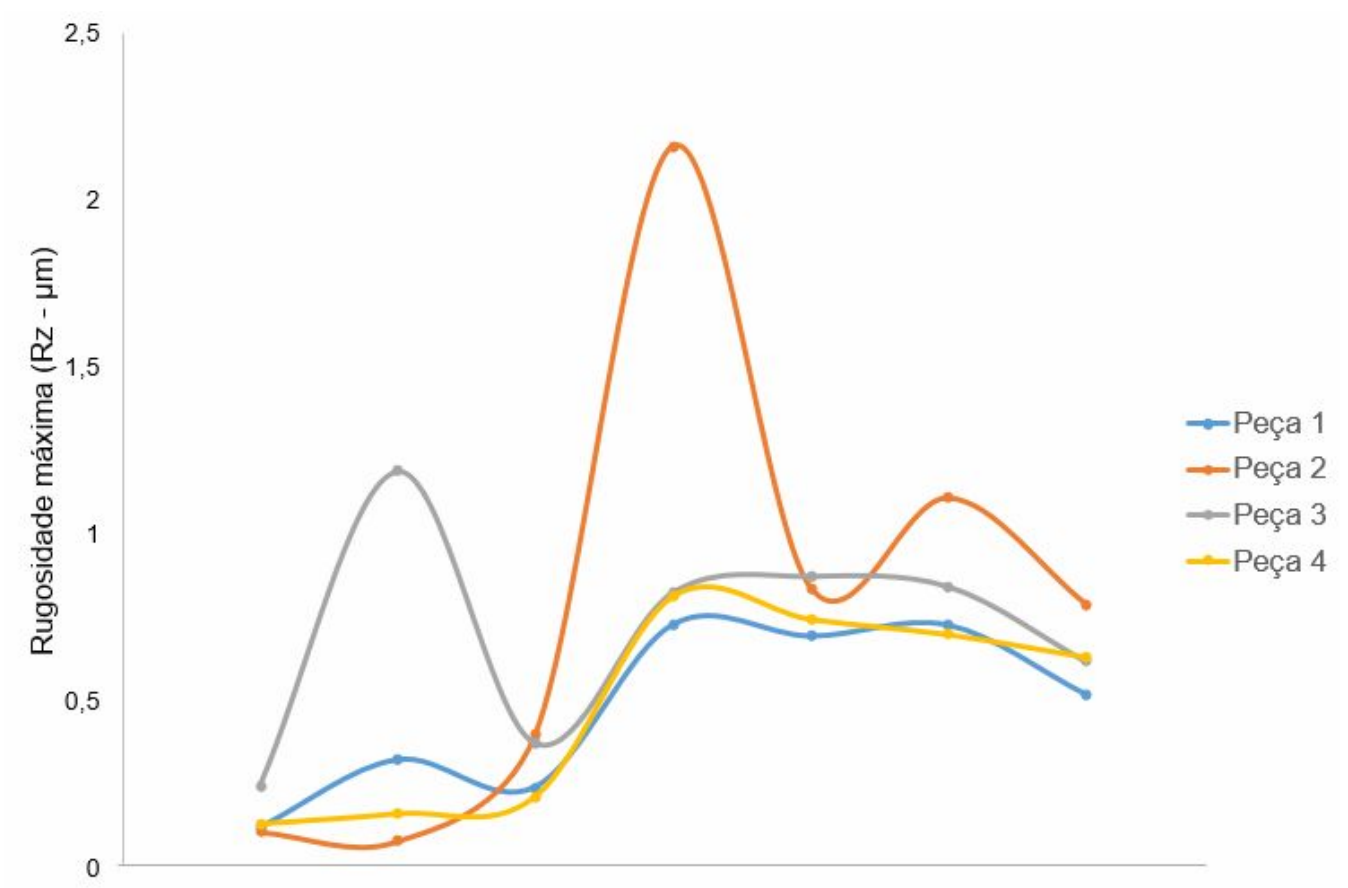

FIGURA 6 Rugosidade máxima de cada uma das peças

O esperado teoricamente, segundo a Equação 1, era que com o aumento do raio de ponta da ferramenta ocorresse a diminuição do parâmetro $R z$, uma vez que $o$ avanço foi mantido constante. No entanto, ao observar a Figura 6 percebe-se que isso só ocorreu para o alumínio. Isso pode ser justificado em função da menor usinabilidade do aço em relação ao alumínio, e também pela atuação dos outros parâmetros inerentes ao processo de torneamento que no aço foram mais significativos do que no alumínio.

Segundo Homami et al. (2014) o acabamento superficial em torneamento é influenciado em vários graus por muitos fatores como taxa de alimentação, velocidade de corte, profundidade de corte, propriedades do material da peça de trabalho, dureza da peça de trabalho, ângulo de aproximação, raio da ponta da ferramenta, tempo de corte, estabilidade da máquina-ferramenta e configuração da peça de trabalho, trepidação e fluidos de corte. Ou seja, a interferência de outros fatores, além do avanço e do raio de ponta da ferramenta, é relatado na literatura, de forma que o aumento da rugosidade mesmo com a elevação do raio de ponta da ferramenta também foi identificado por outros trabalhos.

Gravalos et al. (2010) realizaram um trabalho em que um dos objetivos foi investigar a rugosidade em superfícies usinadas do aço inoxidável superaustenítico ASTM A744 grau CN3MN, de forma que ao aumentarem o raio de ponta da ferramenta utilizada de $0,4 \mathrm{~mm}$ para $1,2 \mathrm{~mm}$ a rugosidade superficial da peça aumentou de 1,04 m para 2,28 $\mathrm{m}$, respectivamente.

Ince e Asiltürk (2015) realizaram um trabalho que apresentava a influência na rugosidade superficial da liga $\mathrm{Co}_{28} \mathrm{Cr}_{6} \mathrm{Mo}$ usinada em um torno $\mathrm{CNC}$ com base nos parâmetros de corte (velocidade de rotação, taxa de avanço, profundidade de corte e raio da ponta). Após avaliação, foi identificado que ao manter fixos o avanço $(0,1$ $\mathrm{mm} / \mathrm{rot})$, a rotação da máquina $(318 \mathrm{rpm})$ e a profundidade de corte $(0,7 \mathrm{~mm})$ e variar os valores do raio de ponta da ferramenta (de $0,8 \mathrm{~mm}$ para $1,2 \mathrm{~mm}$ ) a rugosidade superficial da peça usinada foi de $0,81 \mathrm{~m} \mathrm{e} \mathrm{0,923} \mathrm{m}$, respectivamente. Ao utilizar uma segunda condição de usinagem com avanço $(0,1 \mathrm{~mm} / \mathrm{rot})$, a rotação 
da máquina (636 rpm) e a profundidade de corte $(0,5 \mathrm{~mm})$ fixos, e variando novamente os valores do raio de ponta da ferramenta (de $0,4 \mathrm{~mm}$ para $0,8 \mathrm{~mm}$ ) a rugosidade superficial da peça usinada foi de 1,337 $\mathrm{m} \mathrm{e} \mathrm{1,86} \mathrm{m}$, respectivamente. De forma que, nas duas situações, ao ampliar o valor do raio de ponta da ferramenta, o valor da rugosidade também foi elevado.

Para uma melhor compreensão acerca do comportamento da superfície da peça após o torneamento, a Figura 7 exibe as micrografias de cada uma das superfícies torneadas.

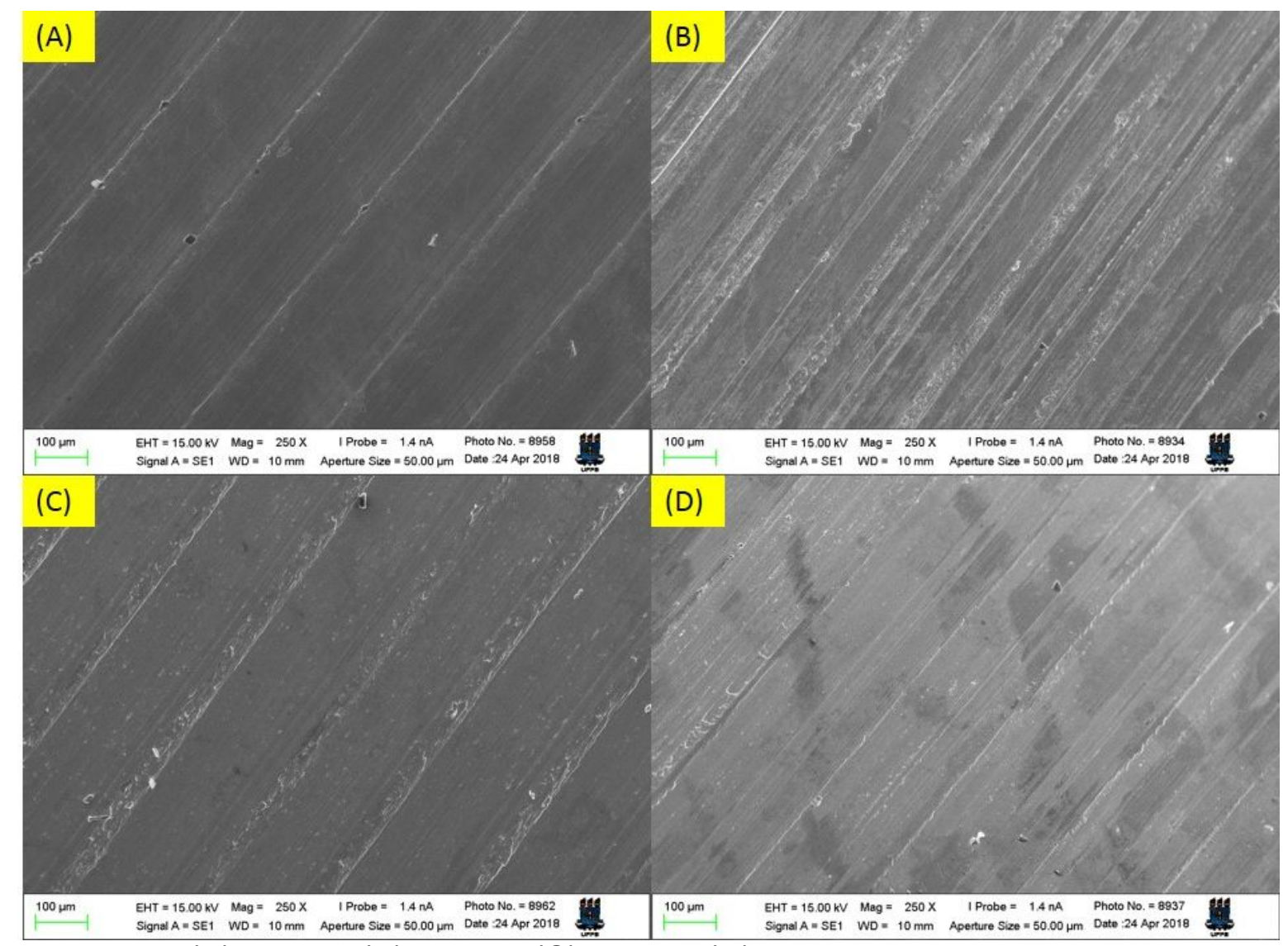

FIGURA 7 (A) Peça 1 (B) Peça 2 (C) Peça 3 (D) Peça 4

Pelas imagens é possível visualizar características comentadas por Cunha e Ribeiro (2003) como marcas de avanço, fluxo lateral, deformações plásticas na superfície e metal aderido à superfície da peça torneada, provenientes do atrito entre a aresta de corte e a superfície usinada.

Além disso, observam-se riscos deixados pela ponta da ferramenta, assim como um aspecto grosseiro resultante do desgaste progressivo da ponta da ferramenta e do aumento da força de corte, decorrente dos passes necessários para remover o sobremetal desejado. Com o desgaste da ferramenta, nota-se o surgimento de possíveis defeitos tais como aglomerados soltos e acumulados de grãos deslocados e arrastados, fazendo com que a superfície apresente um aspecto similar a escamas. Também são perceptíveis pequenas fissuras ao longo da superfície torneada (ASILTURK et al., 2016).

Observa-se que com raio de ponta da ferramenta de $0,4 \mathrm{~mm}$ as superfícies usinadas tanto no aço quanto no alumínio (Peça 1 e 3 , respectivamente) apresentaram uma maior uniformidade no aspecto visual do acabamento. Ao 
estabelecer uma comparação entre as peças de aço (Peças 1 e 2) e as de alumínio (Peças 3 e 4), atenta-se para a existência de uma maior quantidade de falhas e danos superficiais nas peças de aço, uma vez que o alumínio em geral é facilmente usinado, pois durante o torneamento a energia consumida por unidade de volume do metal removido é muito baixa (CUNHA ; RIBEIRO, 2003).

Assim, como no trabalho de Grzesik (2018) e Cunha e Ribeiro (2003), a imagem por MEV permitiu a identificação do avanço adotado, por meio da visualização das marcas deixadas sobre a peça pela ferramenta de corte, como pode ser visualizado na Figura 8, que adotou 0 avanço de 0,22 $\mathrm{mm} / \mathrm{rot} 0$ correspondente a $200 \mathrm{~m}$.

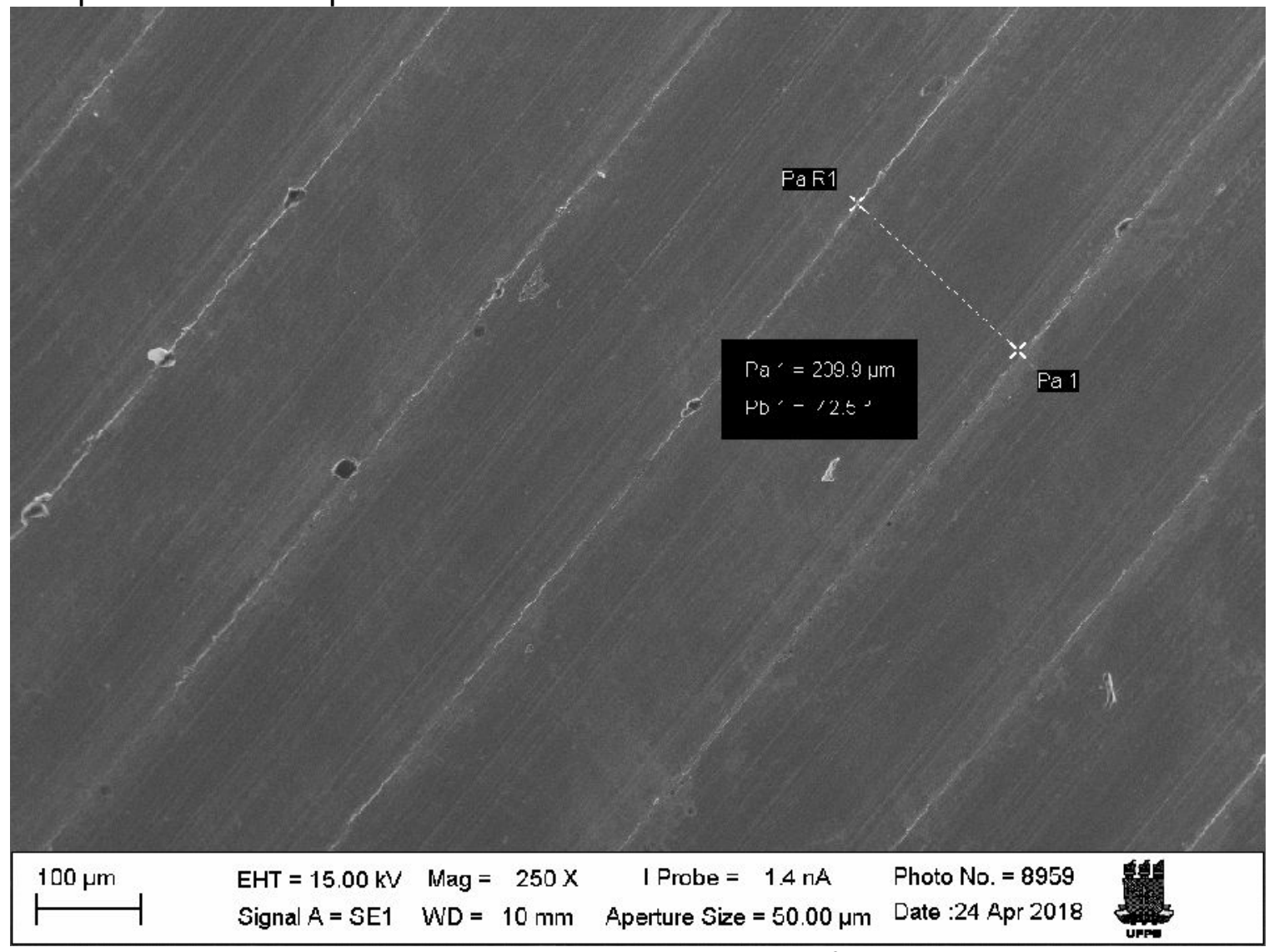

FIGURA 8 Aço torneado com raio de ponta da ferramenta de 0,4 mm

Notam-se que as marcas de avanço na superfície da peça, provenientes da ferramenta de corte, exibem padrões unidirecionais cuja orientação se apresenta perpendicular ao plano de vista, fato esse característico do processo de torneamento (NITHYANANDAM et al., 2015), que também foi observado no trabalho de Das et al. (2016). Como a MEV não permite visualizar a profundidade da superfície. Logo, para uma melhor análise do comportamento superficial das peças torneadas no presente estudo, a Figura 9 ilustra as vistas laterais das peças. 


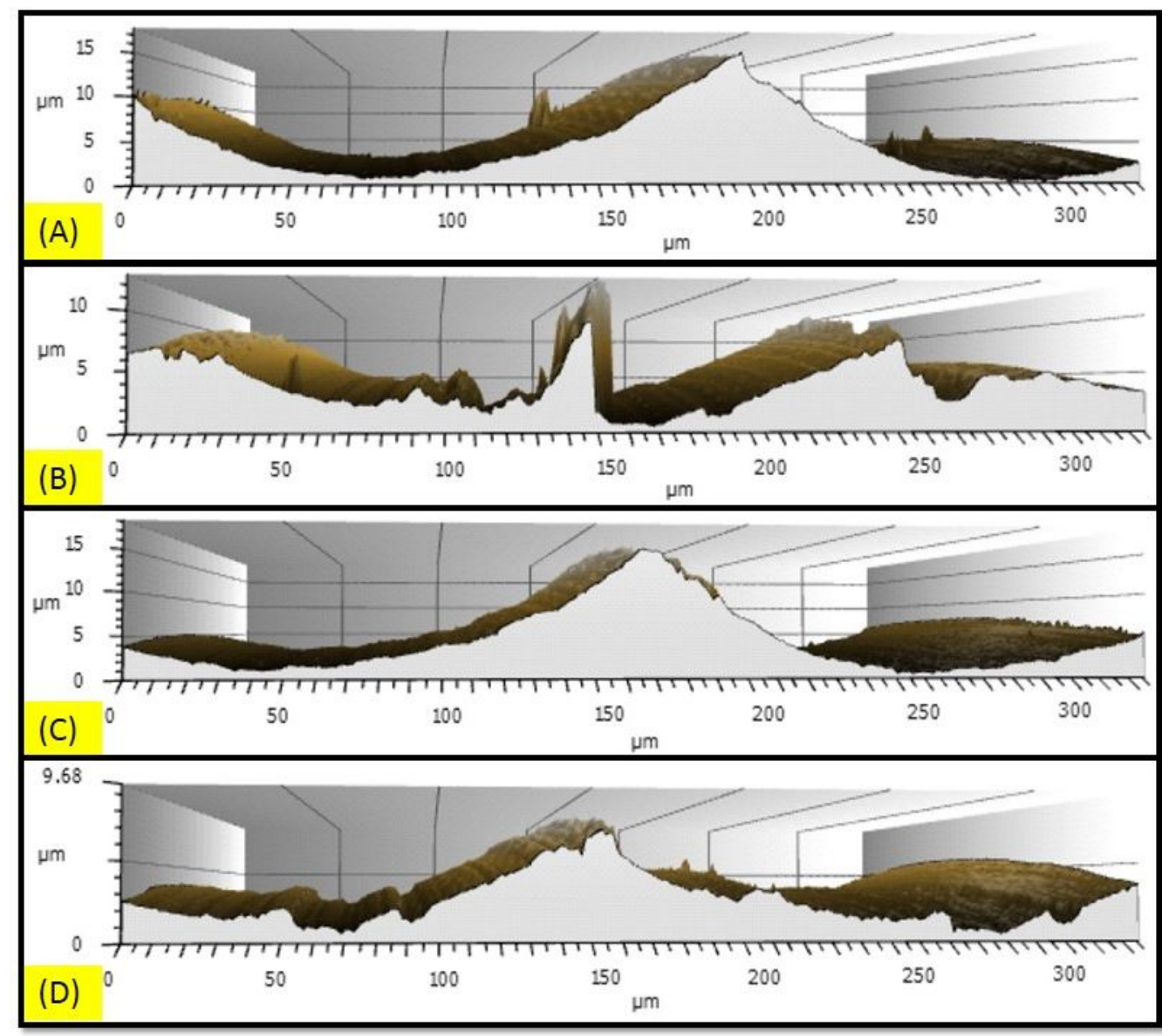

FIGURA 9 Vistas laterais (A) Peça 1 (B) Peça 2 (C) Peça 3 (D) Peça 4

Percebe-se, através da Figura 9 irregularidades superficiais ao longo da peça, e marcas características do raio de ponta da ferramenta, representadas pelos vales da rugosidade, os quais são mais notórios na Peça 3.

\section{CONCLUSÃO}

O presente estudo permitiu identificar que ao aumentar o raio de ponta da ferramenta de $0,4 \mathrm{~mm}$ para $0,8 \mathrm{~mm}$ ocorreu uma diminuição da rugosidade superficial, em termos do parâmetro $\mathrm{Rz}$, quando o material torneado foi o alumínio, concordando com a equação teórica. No entanto, quando o material torneado foi o aço, o inverso ocorreu. Esse fato foi justificado em função da menor usinabilidade do aço e pelos outros parâmetros envolvidos no processo os quais não foram levados em consideração, tais como velocidade de corte, desgaste da ferramenta, atrito e forças de corte.

Além disso, foram obtidas imagens por Microscopia óptica e por perfilometria (vistas laterais) que permitiram identificar as marcas de avanço e do raio de ponta da ferramenta, assim como irregularidades provenientes de fluxo lateral de cavaco, e do próprio atrito peça-ferramenta.

Portanto, foi possível concluir que é necessário o cuidado para garantir a qualidade da superfície da peça e o desgaste lento da ferramenta durante o torneamento. Isso requer otimização de processo para especificar os valores ideais de parâmetros de corte para cada condição de torneamento. 


\section{REFERÊNCIAS}

ASILTÜRK, I.; NEŞELI, S.; INCE, M. A. Optimisation of parameters affecting surface roughness of Co28Cr6Mo medical material during $\mathrm{CNC}$ lathe machining by using the Taguchi and RSM methods. Measurement, v. 78, p. 120-128, 2016. Disponível em: $<$ https://www.sciencedirect.com/science/article/pii/S0263224115005217>. DOI: 10.1016/j.measurement.2015.09.052.

ABNT - Associação Brasileira de Normas Técnicas . NBR ISO 4287: Especificações geométricas do produto (GPS) - Rugosidade: Método do perfil - Termos, definições e parâmetros da rugosidade. Rio de Janeiro, 2002.

BHARDWAJ, B.; KUMAR, R.; SINGH, P. K. Prediction of surface roughness in turning of EN 353 using response surface methodology. Transactions of the Indian Institute of Metals, v. 67, n. 3, p. 305-313, 2014. Disponível em: <https://link.springer.com/article/10.1007/s12666-013-0346-7>. DOI: 10.1007/s12666-013-0346-7.

CUNHA, E. A.; RIBEIRO, M. V. Influência da velocidade de corte na rugosidade em usinagem de ligas de alumínio. In: Congresso Brasileiro de Engenharia de Fabricação, 2., 2003, Uberlândia. Anais do II COBEF, 2003.

DAS, S. R.; KUMAR, A.; DHUPAL, D. Experimental investigation on cutting force and surface roughness in machining of hardened AISI 52100 steel using cBN tool. International Journal of Machining and Machinability of Materials, v. 18, n. 5-6, p. 501-521, 2016. Disponível em: < https://www.inderscienceonline.com/doi/abs/10.1504/IJMMM.2016.078997>. DOI: 10.1504/IJMMM.2016.078997.

FERRARESI, D. Usinagem dos metais. Editôra Edgard Blücher, 2014.

GILLIBRAND, D.; SARWAR, M.; PIERCE, C. T. The economic benefit of finish turning with coated carbide. Surface and Coatings Technology, v. 86, p. 809-813, 1996.

Disponível em: https://www.sciencedirect.com/science/article/pii/S0257897296030599>. DOI: 10.1016/S0257-8972(96)03059-9.

GRAVALOS, M. T.; MARTINS, M.; DINIZ, A. E.; MEI, P. R. Influência da rugosidade na resistência à corrosão por pite em peças torneadas de aço inoxidável superaustenítico. Rem: Revista Escola de Minas, v. 63, n. 1, 2010. Disponível em: <http://www.redalyc.org/html/564/56416597013/>.

GRZESIK, W. Prediction of surface topography in precision hard machining based on modelling of the generation mechanisms resulting from a variable feed rate. The International Journal of Advanced Manufacturing Technology, v. 94, n. 9-12, p. 4115-4123, 2018. Disponível em: <https://link.springer.com/article/10.1007/s00170017-1129-9>. DOI: https://link.springer.com/article/10.1007/s00170-017-1129-9.

HOMAMI, R. M.; TEHRANI, A. F.; MIRZADEH, H.; MOVAHEDI, B.; AZIMIFAR, F. Optimization of turning process using artificial intelligence technology. The 
International Journal of Advanced Manufacturing Technology, v. 70, n. 5-8, p. 1205-1217, 2014.

HORVÁTH, R.; CZIFRA, A.; DRÉGELYI-KISS, A. Effect of conventional and nonconventional tool geometries to skewness and kurtosis of surface roughness in case of fine turning of aluminium alloys with diamond tools. The International Journal of Advanced Manufacturing Technology, v. 78, n. 1-4, p. 297-304, 2015. Disponível em: < https://link.springer.com/article/10.1007/s00170-014-6642-5>. DOI: $10.1007 / \mathrm{s} 00170-014-6642-5$

INCE, M.; ASILTÜRK, I. Effects of cutting tool parameters on surface roughness. International Refereed Journal of Engineering and Science, v. 4, n. 8, p. 15-22, 2015. Disponível em: <http://www.irjes.com/Papers/vol4issue8/C481522.pdf $>$.

KUMAR, S.; SINGH, D.; KALSI, N. S. Analysis of surface roughness during machining of hardened AISI 4340 steel using minimum quantity lubrication. Materials Today: Proceedings, v. 4, n. 2, p. 3627-3635, 2017. Disponível em: < https://www.sciencedirect.com/science/article/pii/S2214785317304650 >. DOI: 10.1016/j.matpr.2017.02.255

MAITY, K.; PRADHAN, S. Investigation of tool wear and surface roughness on machining of titanium alloy with MT-CVD cutting tool. In: IOP Conference Series: Materials Science and Engineering. IOP Publishing, 2018. p. 012053. Disponível em: < http://iopscience.iop.org/article/10.1088/1757-899X/346/1/012053/meta>. DOI: 10.1088/1757-899X/346/1/012053.

NITHYANANDAM, J.; DAS, S. L.; PALANIKUMAR, K. Influence of cutting parameters in machining of titanium alloy. Indian Journal of Science and Technology, v. 8, n. S8, $\quad$ p. 556-562, 2015. Disponível em: < http://www.indjst.org/index.php/indjst/article/view/71291>. DOI: 10.17485/ijst/2015/v8iS8/71291.

QEHAJA, N.; JAKUPI, K.; BUNJAKU, A.; BRUÇI, M.; OSMANI, H. Effect of machining parameters and machining time on surface roughness in dry turning process. Procedia engineering, v. 100, p. 135-140, 2015. Disponível em: < https://www.sciencedirect.com/science/article/pii/S1877705815003781>. DOI: 10.1016/j.proeng.2015.01.351.

SOUZA, M. S.; LAURO, C. H.; BRANDÃO, L. C. Análise de rugosidade em função da velocidade de avanço em operações de torneamento. In: Congresso Nacional de Engenharia Mecânica, 6., 2010, Campina Grande. Anais do VI CONEM, 2010. 